\title{
Precise bonding-free micromoulding of miniaturized elastic inflatable actuators
}

\author{
Edoardo Milana ${ }^{1}$, Mattia Bellotti ${ }^{1}$, Benjamin Gorissen ${ }^{1}$, Michaël De Volder ${ }^{1,2}$ and Dominiek Reynaerts ${ }^{1}$
}

\begin{abstract}
A new precise micromoulding process has been developed in order to fabricate elastic inflatable microactuators, having a corrugated inner cavity without requiring any additional bonding step. Micromoulds are machined using micromilling and wire electrical discharge grinding. This manufacturing process allows the out-of-plane fabrication of complex shapes of microactuators with smallest feature down to $40 \mu \mathrm{m}$. It is possible to fabricate miniaturized versions of actuators that are normally made at a larger scale. Particularly, this paper reports a silicone-made miniaturized elastic inflatable actuator showing a large bending deformation. The particular pattern of the inner cavity forms a corrugated structure and enables a fully curled deformation of the actuator due to the equal distribution of multiple bending points. As a demonstrator, three microactuators are used as active fingers of a soft microgripper.
\end{abstract}

\section{INTRODUCTION}

Compared to rigid robots, a clear advantage of using soft robots is their safe interaction with its surroundings due the used compliant structures and materials [1]. This intrinsic compliancy of soft robots is transformed into the stunning property of adaptability. When in touch with external bodies, from animals to more rigid object, soft robots adapt to the encountered shape, which makes them suitable to work in unstructured environments [2]. In this context, the tasks that soft robots can perform vary from grasping unknown-shaped objects to circumventing unpredicted obstacles.

Regarding soft grippers, Shintake et al. [3] recently reviewed the different technologies in literature, distinguishing them according to actuation principles, controlled stiffness and controlled adhesion. One type of grippers, based on elastic inflatable actuators (EIAs), have gotten particular traction in soft robotic literature [4] and are now even becoming commercially available [5].

On the other side of the application spectrum, researchers proposed soft robots which are able to overcome obstacles, such as a walking robot that crawls underneath an obstruction [6], an inflatable growing soft vessel which navigates through the environment [7], soft deformable origami wheels that allow a vehicle to adapt its shape to the road [8]. However, specific applications necessitate a downscaling of actuator technology.

*This research is supported by the Fund for Scientific Research-Flanders (FWO), and the European Research Council (ERC starting grant HIENA).

${ }^{1}$ Edoardo Milana, Benjamin Gorissen, Michaël De Volder and Dominiek Reynaerts are with the Department of Mechanical Engineering, KU Leuven, Members Flanders Make, Leuven, Belgium edoardo.milana@kuleuven.be

${ }^{2}$ Michaël De Volder is also with the Institute for Manufacturing, Dept. of Engineering University of Cambridge Cambridge, UK
For example, in minimally invasive surgery, miniaturized soft robotic actuators perform delicate and ultraprecise tasks, where the soft robotic tools need to be flexible and able to navigate inside a human body without damaging it [9], which is nearly impossible to achieve using rigid robotics. Other examples in this domain include active catheters [10] and chip on tip endoscopes [11], that are able to move through natural orifices of only a few millimeters in size. Similarly, it is anticipated that a miniaturization of soft inflatable grippers to the millimeter or sub-millimeter domain enables versatile manipulation of small-scale objects of irregular shape, texture and stiffness. However, as large scale EIAs in literature typically have an intricate design [4], miniaturization into the millimeter range is not straightforward. Manufacturing processes cannot be directly downscaled, and therefore previous research focused on miniaturizing simplified designs [12], losing overall performance.

In this paper we present a novel micromanufacturing technique which improves the state of the art and allows the fabrication of more complex and better performant actuators. Among all types of sub-centimeter soft actuators, reviewed by Hines et al. [13], we focus on bending elastic inflatable microactuators, which find application in micromanipulators [14], active catheters [10] and biomimetic ciliary propulsion [15]. Those actuators were first introduced by Suzumori [16] and Konishi [17] and consist of cantilever structures, typically made of PDMS or other rubbers, with an internal void, for such reason also called pneumatic balloon microactuators. This void causes the actuator to bend once pressurized with air, due to its asymmetric positioning with respect to the symmetry axis of the cantilever.

As their counterpart at a larger scale, those actuator are typically manufactured in two different parts that are then bonded together to seal the inner void [18]. The bonding step at a millimeter scale is more difficult, as it requires a precise alignment system for the two parts, which make further miniaturization very challenging. Moreover, the rupture of the actuators mainly occur in the bonded line, decreasing the mechanical performances. To solve this issue, Gorissen et al. [12] proposed a bonding-free process where cylindrical actuators are made in a single step by using out-of-plane high aspect ratio moulding, which allow to obtain dimensions of $1 \mathrm{~mm}$ diameter and $10 \mathrm{~mm}$ length. Cylindrical microrods are inserted in a micromilled mould to give the negative shape of the inner void. A further miniaturization of the same design was reported by Paek [19], who manufactured a microtentacle with diameter $0.15 \mathrm{~mm}$ and length $5-8 \mathrm{~mm}$, using a direct peeling-based bonding-free technique. 


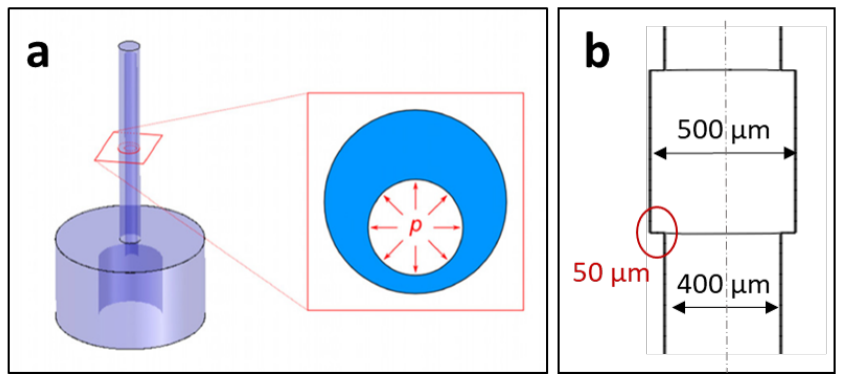

Fig. 1. a) 3-D representation of a soft bending microactuator with a cylindrical inner void. b) Profile sketch of a patterned inner cavity with $50 \mu \mathrm{m}$ grooves.

These processes increase the mechanical robustness as the actuator is a single monolithic piece, but they are limited to the full cylindrical shape of the inner chamber, while corrugated and bellows shapes are normally integrated in the design of EIAs due to their amplification or modification of the bending motion [13].

This paper reports on a new manufacturing process based on wire electrical discharge grinding (WEDG) to introduce corrugation in the cylindrical inner chamber of elastic inflatable microactuators compatibly with the bonding-free process developed by Gorissen. While WEDG has already been used to manufacture pneumatic piston-cylinder microactuators [20], [21], in this case it is used to machine the cylindrical microrods and obtain the desired corrugated shape, which is then replicated via micromoulding in the inner chamber of the microactuators.

In the first section, a design compatible with the fabrication process is presented. FEM simulations are carried out to compare variations of this design to the fully cylindrical actuator shape that are found in literature, showing the mechanical advantages of introducing corrugated profiles. Subsequently, the manufacturing process is described. A particular focus is given to the WEDG technique. Further, the new microactuator is tested and characterized. In conclusion, three microactuators are used to build a microgripper as demonstrator.

\section{DESign And FEM Simulations}

An elastic inflatable microactuator, manufactured through a bonding-free process, typically consists of a cylindrical beam having an eccentric cylindrical inner cavity, as depicted in Fig.1a. Pressurization of the inner cavity deforms the beam into a bending configuration. However, previous works have shown that there is a limit to the achievable curvature radius after which the microactuator starts expanding until it eventually bursts [12]. On contrary, soft bending actuators with corrugated inner voids, also called PneuNets [22], show both at the macro and micro scales a high degree of bending, causing the structure to fully curl and enabling grasping applications. In order to realize this corrugated inner chamber, these actuators are typically made out of two parts bonded together with an additional manufacturing step. This causes a weakening of the mechanical properties of the structure, resulting in lower attainable pressures. Moreover, for miniaturized soft actuators the bonding step becomes
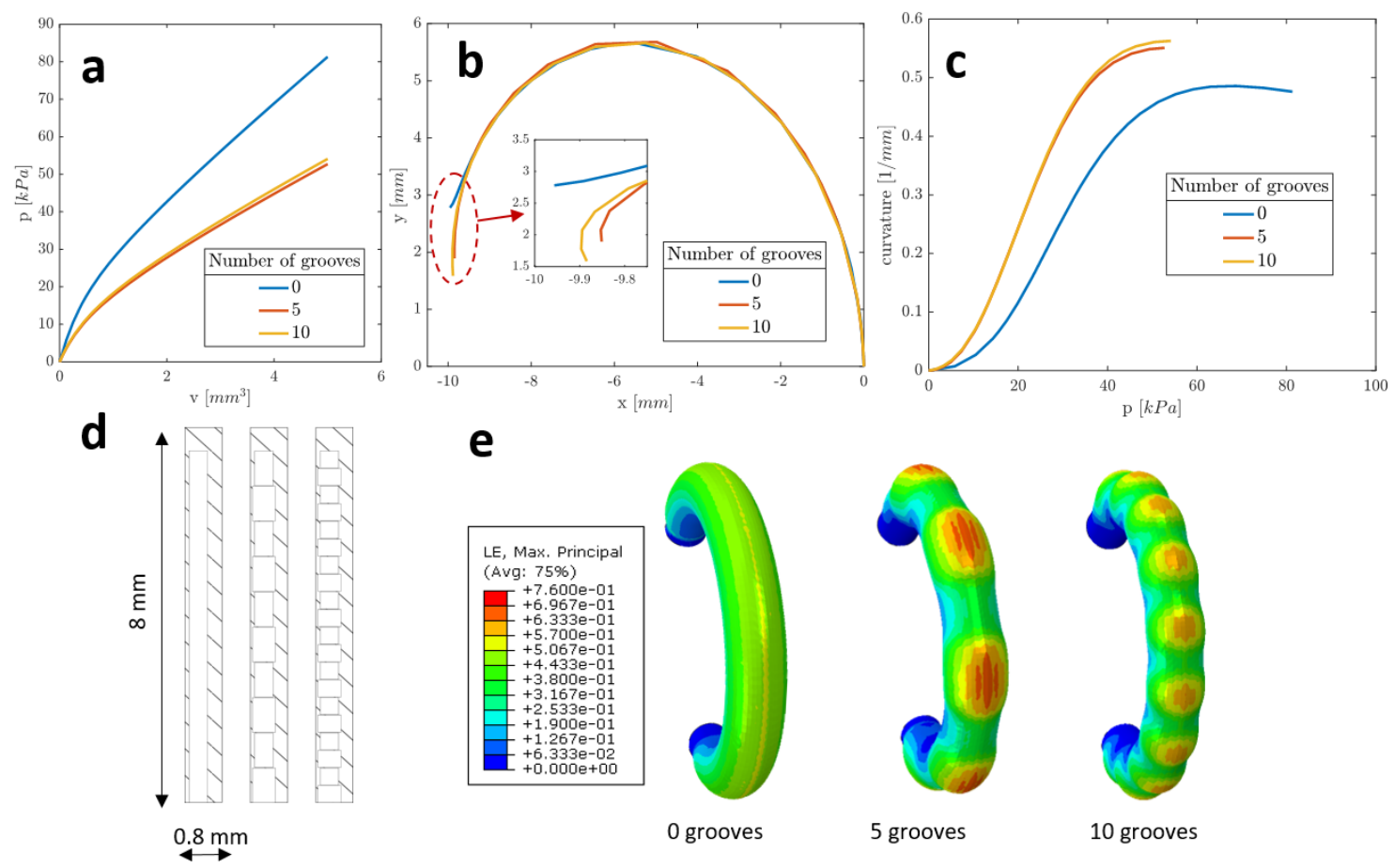

Fig. 2. FEM simulation comparison between three microactuators having 0,5 and 10 grooves in their inner void. a) Pressure-Volume curves. b) Tip trajectories. c) Bending curvature vs. pressure. d) Section view of the three actuators. e) Logarithmic strain distribution for the three actuators at the same bending radius 
very challenging and decreases the level of precision in the manufacturing process. This highlights the need of introducing corrugated shape in the inner chamber of microactuators compatibly with the bonding-free process.

Simple cylindrical microactuators are made with a twopart micromilled mould where a cylindrical microrod is positioned as insert into the mould to provide the shape of the inner cavity. After the polymerization of silicone rubber, the microactuator is demoulded and the microrod is removed from the bottom, resulting in an open cavity. This process allows the production of microactuators with a length of 8 $\mathrm{mm}, 0.8 \mathrm{~mm}$ diameter and $0.11 \mathrm{~mm}$ eccentricity of the inner cavity.

The simplest way of introducing membrane corrugation is to pattern the profile of the inner cavity with a certain number of equally-spaced grooves of the same depth. Such grooves are machined into the microrod and replicated in the inner cavity of the microactuator. The first technical consideration in the design phase is the fact that the microrod needs to be removed from the microactuator, limiting the depth of the groove. A deep groove may prevent the microrod removal without damaging the actuator. However, thanks to the high deformability of silicone rubber, this problem is not a concern with thin grooves. For these reasons, we fixed the groove depth to $50 \mu \mathrm{m}$, patterning the inner cavity by alternating the diameter between 500 and $400 \mu \mathrm{m}$ (Fig. 1b).

To evaluate the deformation of this new design of soft microactuators, we performed static nonlinear FEM simulations using the commercial code ABAQUS. By using the fluid-structure interaction tool [23], the cavity is modeled as initially filled with fluid and the inflation is simulated as an increase of the volume enclosed in the cavity. This approach makes it possible to monitor pressure and volume of the microactuator at each step and avoid possible convergence problems caused by the nonlinearities of the pressure-volume curve. As the microactuators are made out of Dragon Skin 30, an Ogden model $(\mathrm{G}=75.5 \mathrm{kPa},=5.84$ [24]) is used to describe the hyperelastic mechanical properties.

In a first comparison we studied the impact of a different number of grooves $(0,5,10)$ equally distributed along the length of the inner cavity as shown in Fig.2d. A number of grooves equal to 0 corresponds to the fully cylindrical shape of the inner cavity. It is possible to notice not only how the pressure required to bend the actuator drastically diminishes by introducing grooves (subfigure a and c), but also how the tip trajectory diverges from bending after a certain radius for a cylindrical cavity. On the contrary, the tip continues to curl for corrugated cavities, even further for the higher number of grooves (inset figure b). In Fig. 2e the distribution of the logarithmic strains are reported for the same bending radius of the three actuators. The introduction of the grooves locally increases the strains because it forms a pattern of alternate membrane thicknesses, where the thinnest inflate more than their thicker neighboring, due to the less amount of material. This different local inflation causes the microactuator section corresponding to the thin membranes to bend more. Thus, the deformation is not constant all
TABLE I

ELASTIC INFLATABLE MICROACTUATOR DIMENSIONS

\begin{tabular}{cc} 
Feature & Values $(\mathbf{m m})$ \\
\hline Actuator length $(L)$ & 8 \\
Actuator diameter $(D)$ & 0.8 \\
Eccentricity $(e)$ & 0.11 \\
Inner cavity length $(l)$ & 7.5 \\
Inner cavity large diameter $\left(d_{1}\right)$ & 0.5 \\
Inner cavity large diameter $\left(d_{2}\right)$ & 0.4 \\
Thin membrane length $(a)$ & 0.4 \\
Thick membrane length $(b)$ & 0.35 \\
\hline
\end{tabular}

over the structure but it is distributed, leading to the formation of discrete bending section, which behave similarly to compliant hinges. It clearly emerges from this analysis how the introduction of grooves reduces the load pressures and enhances the bending performance of microactuators. The two corrugated actuators perform similarly due to the same symmetric alternate pattern. However, the actuator with 5 grooves shows a more segmented deformed configuration due to the higher bending localization, which explains the reason why it undergoes larger strains than the 10-grooves actuator. The maximum logarithmic strain is 0.76 , which corresponds to an engineering strain of $114 \%$, below the maximum elongation at break of Dragon Skin $30(364 \%)$ [25], but still a reason of concern regarding the fatigue life of the actuator. A change in the morphology of the 5-grooved actuator by replacing the alternate symmetric pattern with a reduction of the groove length would reduce the maximum strains, but increase the bending localization. The resulting discrete distribution of bending points would be ideal for a microfinger rather than for a fully curled actuator. Thus, we opted for manufacturing the microactuator with 10 grooves. Table 1 reports the dimensions of the actuator.

\section{SOFT ACTUATOR MANUFACTURING}

The corrugated microactuator is manufactured with a bonding-free, out of plane high aspect ratio moulding. A

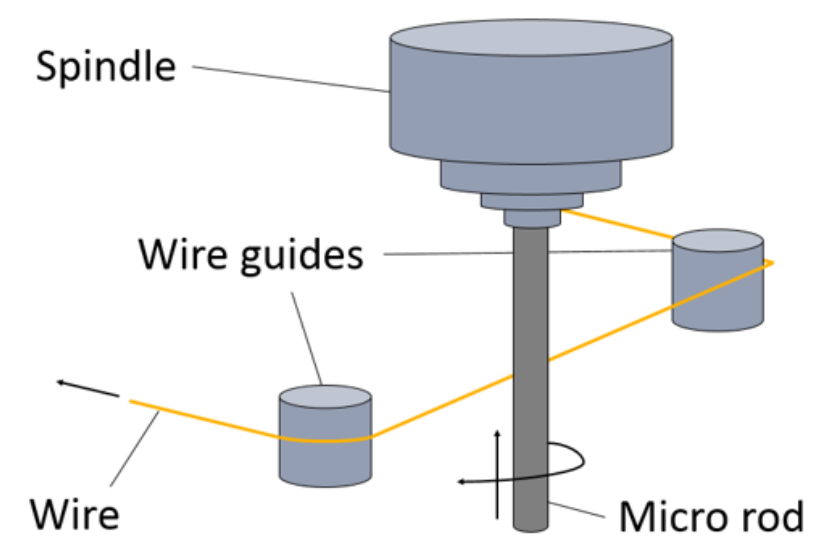

Fig. 3. Schematic of WEDG processing of the micro rods. 

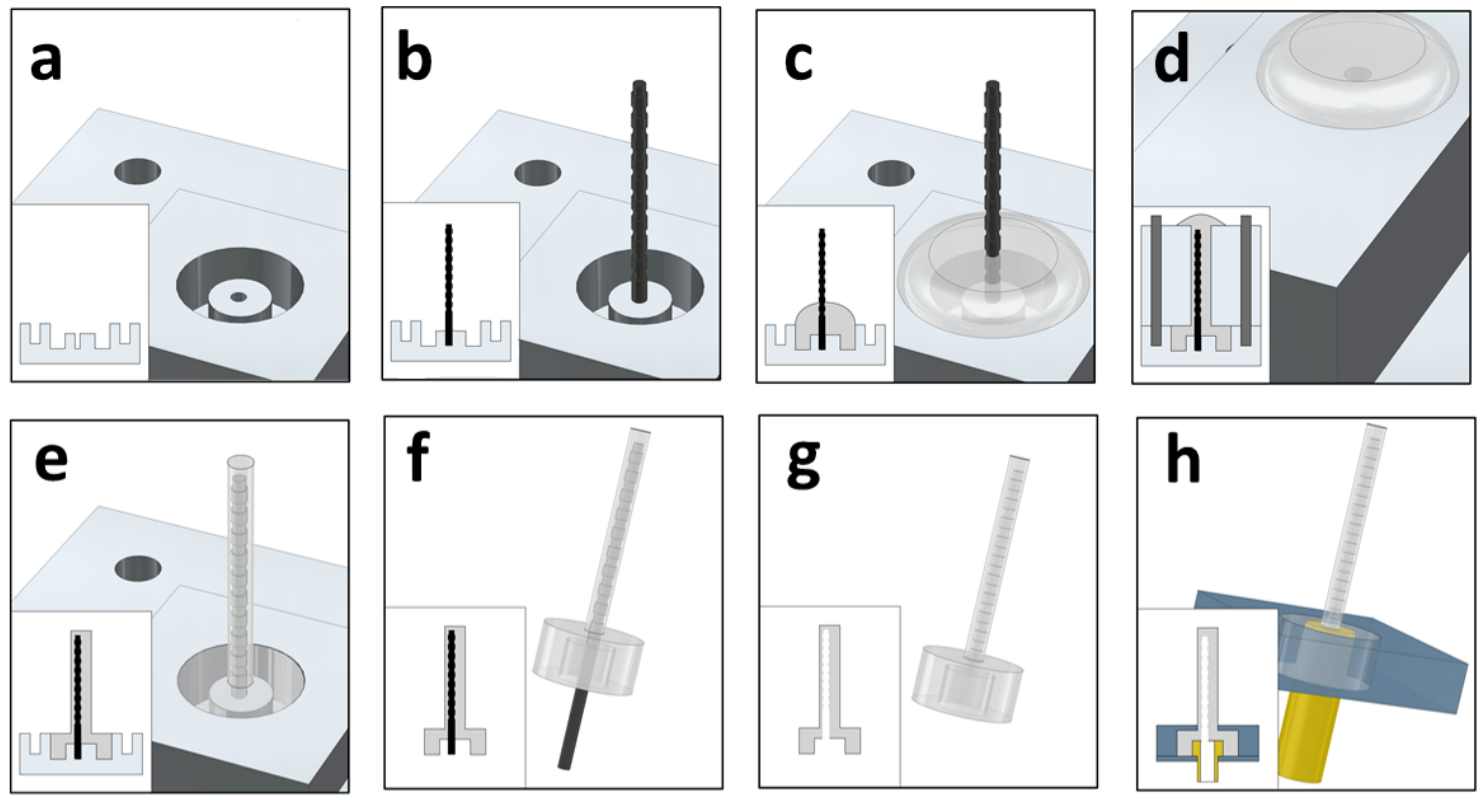

Fig. 4. Micromoulding process 3D view and section view in the insets. a) Bottom part of the mould. b) Placing microrod in the designated cavity. c) Pouring liquid silicone elastomer. d) Aligning the top part and closing the mould. e) Curing the elastomer and opening the mould. f) Demoulding the microactuator. g) Removing the microrod. h) Connecting and sealing the microactuator

two-part mould is made out of aluminium using a 5-axis micro-milling machine (Kern MMP). The corrugated shape of the inner cavity is replicated on a microrod by means of an in-house developed WEDG process, described in the following section. The absence of the bonding step increases the manufacturing precision.

\section{A. Microrod machining}

The microrods are machined using wire electrical discharge grinding (WEDG) [26]. In this process, material is removed through melting and vaporisation from a rotating cylindrical microrod using a continuously-fed wire by means of sequences of high frequency sparks $(50-150 \mathrm{kHz})$ in presence of a dielectric medium (Fig. 3). Theoretically, WEDG can be used to reduce the section of the microrods down to $10 \mu \mathrm{m}$ diameter [27]. However, as already mentioned in the previous paragraph, in our applications significant reductions of the section would prohibit the demoulding of the actuators. This explains why we opted for a depth of the grooves limited to $50 \mu \mathrm{m}$, even though this could be increased accordingly to the mechanical properties of the rubber. The WEDG unit of a SARIX SX-100-HPM micromachining tool is used. Tungsten carbide cylindrical rods of $500 \mu \mathrm{m}$ nominal diameter are chosen because of the high stiffness of tungsten carbide, roughly double than steel. A brass wire of $200 \mu \mathrm{m}$ diameter is used. Hydrocarbon oil (HEDMA 111) is applied as dielectric fluid. In order to reduce the machining time, WEDG processing is carried out through two regimes: roughing and finishing. In roughing regime a relatively high energy input per discharge is applied to maximize the material removal rate. On the contrary, a lower energy input per discharge is applied during finishing regime. This is done by lowering the values of the process parameters determining the discharge energy (i.e. open voltage, capacitance, and pulse duration). In this way, microrods having a relatively low surface roughness ( $\mathrm{Sa}$ $=0.37 \mu \mathrm{m}$ ) and acceptable process repeatability (standard deviation of groove depth $=2.67 \mu \mathrm{m}$ ) are obtained [28]. The process parameters applied in each regime are summarised in Table 2. The time required to machine a microrod varies from approximately 40 minutes ( 5 grooves) to 80 minutes (10 grooves) when applying these parameters.

\section{B. Micromoulding}

Fig. 4 displays the sequence of the total moulding process. Firstly, the machined microrod is inserted in the designated place in the bottom part of the mould (Fig. 4b) and a releasing agent (Devcon) is applied on the functional surfaces. Then, the two pre-components of Dragon Skin 30 are mixed in a ratio 1:1, degassed and poured on the bottom part of the mould (Fig. 4c). An additional degassing step can be made to definitely eliminate air bubbles trapped during pouring. By means of $3 \mathrm{~mm}$ alignment pins, the top part is aligned on the

TABLE II

APPLIED PROCESS PARAMETERS DURING WEDG

\begin{tabular}{ccc} 
Parameter & Roughing & Finishing \\
\hline Open Voltage $u_{0}[\mathrm{~V}]$ & 120 & 85 \\
Capacitance $C[\mathrm{nF}]$ & 5 & 1.5 \\
Pulse duration $T_{O N}[\mathrm{~s}]$ & 5 & 4 \\
Pulse interval $T_{O F F}[\mathrm{~s}]$ & 3 & 2 \\
Spindle rotation $[\mathrm{rev} / \mathrm{min}]$ & 850 & 700 \\
Depth of cut $a_{p}[\mu \mathrm{m}]$ & 20 & 10 \\
\hline
\end{tabular}




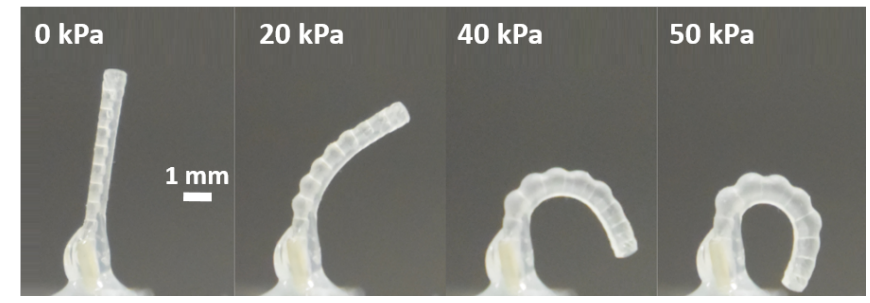

Fig. 5. Deformed configuration of the microactuator at different pressures.

bottom part and placed in contact. In order to have a precise control over the manufacturing process and small tolerances on the final microactuator, a location fit $\mathrm{H} 7 / \mathrm{h} 6$ is used for the alignment pins. To make sure the liquid rubber fills all the gaps, the two parts are tightened with screws, letting the extra rubber coming out on the top part surface (Fig. 4d). The mould is placed in the oven at $60{ }^{\circ} \mathrm{C}$ for 1 hour to cure the rubber. Afterwards, extra Dragon Skin is removed from the top part and the mould is opened, using ethanol as lubricant (Fig. 4e). Subsequently, the microactuator is removed from the mould, while the microrod is still inside the cavity (Fig. 4f). With the help of tweezers the microrod is gently removed from the microactuator (Fig. 4g), which is eventually connected to the pressure source (Fig. 4h).

\section{EXPERIMENTAL}

\section{A. Microactuator testing}

The microactuator is clamped and tested using a pressurecontrol setup, where different load pressures are applied and camera images of the deformed configurations are taken. Fig. 5 shows the actuator deformation at 4 different pressure values. The highest functional bended configuration is achieved at $50 \mathrm{kPa}$, making it possible to use those microactuators for grasping small objects. At higher pressures the actuator tip touches its body and eventually the extra inflation of the thinnest membranes makes the actuator bursts. Compared to the FEM simulation, where a uniform inflation of the membranes is seen, we observed a certain inflation sequence starting from the bottom of the actuator till the tip. As such, the bending motion propagates along the structure during the actuation, instead of having a constant bending radius.
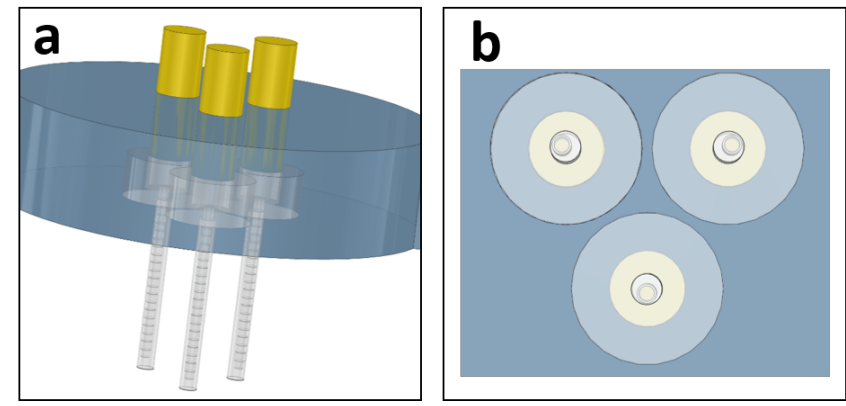

Fig. 6. a) 3-D sketch of the microgripper. b) bottom view of the microgripper

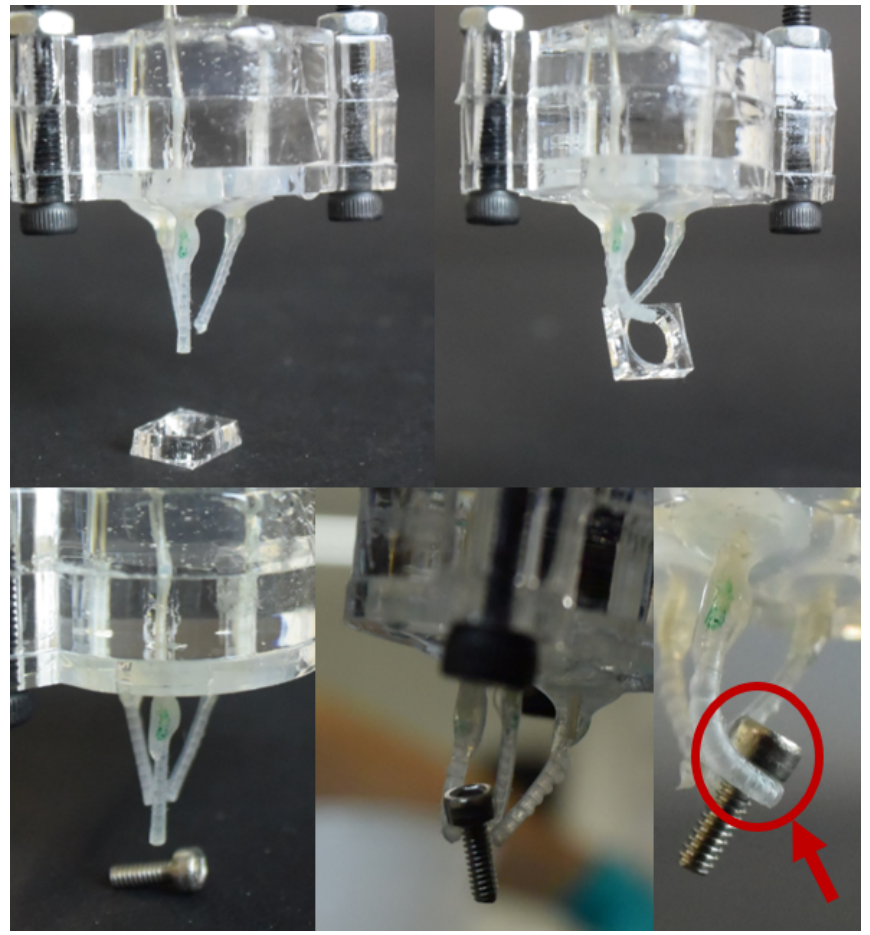

Fig. 7. Pictures of the operational modes of the microgripper. In the top pictures the microgripper is grasping a $2 \times 6 \times 6 \mathrm{~mm}$ Plexiglas component and in the bottom pictures a M1.6x5 screw. Highlighted and pointed out in red the adaptive properties discussed in the text.

Probably, a slight misalignment between the microrod and the vertical wall of the top mould caused a variation of the membranes thicknesses. However, it is interesting to note how the previous design reported in literature [12] could not achieve such a level of deformation, confirming the importance of the corrugated inner cavity.

\section{B. Microgripper}

To demonstrate the applicative possibilities of our technology, we assembled three equal microactuators to form a soft microgripper, as shown schematically on Fig. 6. Three actuators are placed in parallel with an angle of $120^{\circ}$ as depicted in subfigure $b$. The gripping performance of this device is shown on Fig. 7. As displayed in the pictures, the microgripper can grasp different objects of different materials and shapes, due to the compliancy of the soft structure. In the subfigures, as examples, the microgripper is grasping a 2x6x6 mm component of Plexiglas and M1.6x5 steel screw. Particularly it is possible to observe a significant example of how the soft actuatotors adapt to the grasped object when grasping the small screw (red arrow in subfigure). When in touch with the screw, although the inner cavity is pressurized, the bending deformation moves out of plane to allow the microactuator to wrap around the screw to have a conformal grip.

\section{Conclusion}

A new micromanufacturing technique has been developed to increase the design possibilities of bonding-free made soft 
microactuators. Through wire electrical discharge grinding of microrods, we were able to machine very small patterns (down to $50 \mu \mathrm{m}$ ) on a mould. This pattern is replicated in the inner cavity of soft microactuators, through an out-of-plane moulding process. This technique allows a precise manufacturing of a monolithical small-scale soft robotic structures, $8 \mathrm{~mm}$ long and $0.8 \mathrm{~mm}$ in diameter, without the need of a bonding step between different rubber parts, avoiding the mechanical weaknesses caused by the bonding and enabling a precise control on the manufacturing parameters.

Moreover, compared to previous designs used for the bonding-free process, which contemplated a simple cylindrical inner cavity, the introduction of corrugated shapes made it possible to achieve larger bending deformations, similar to the motion observed in larger-scales PneuNets bending actuators. The previous design was adopted to create array of artificial pneumatic cilia for biomimetic fluid propulsion, and, in this context, the new microactuator could be applied more efficiently due to the larger bending motion. Furthermore, by integrating three soft microactuators into a microgripper, we were able to pick up small objects of different shapes and materials.

WEDG is a versatile technique that allows to machine not only linearly-spaced grooves but also more complex shapes of microrods, such as nonlinear asymmetric patterns or conical shapes. Thus, the main limitation of this technique lies in the moulding process of the actuator, particularly on extracting the microrod without damaging the actuator. Despite this technical limitation, there are still plenty of design possibilities to explore with our technique, such as actuators with localised bending points for microfingers manufacturing, controlled sequential inflation or bi-modal deformation.

\section{ACKNOWLEDGMENT}

The authors would like to thank Shashwat Kushwaha for micromilling the mould.

\section{REFERENCES}

[1] S. Kim, C. Laschi, and B. Trimmer, "Soft robotics: A bioinspired evolution in robotics," Trends Biotechnol., vol. 31, no. 5, pp. 287294, 2013.

[2] F. Iida and C. Laschi, "Soft Robotics: Challenges and Perspectives," Procedia Comput. Sci., vol. 7, pp. 99-102, 2011.

[3] J. Shintake, V. Cacucciolo, D. Floreano, and H. Shea, "Soft Robotic Grippers," Adv. Mater., p. 1707035, may 2018.

[4] B. Gorissen, D. Reynaerts, S. Konishi, K. Yoshida, J.-W. Kim, and M. De Volder, "Elastic Inflatable Actuators for Soft Robotic Applications," Adv. Mater., p. 1604977, sep 2017.

[5] "Soft Robotics Inc., https://www.softroboticsinc. com."

[6] R. F. Shepherd, F. Ilievski, W. Choi, S. A. Morin, A. A. Stokes, A. D. Mazzeo, X. Chen, M. Wang, and G. M. Whitesides, "Multigait soft robot," Proc. Natl. Acad. Sci., vol. 108, no. 51, pp. 20400-20403, 2011.

[7] E. W. Hawkes, L. H. Blumenschein, J. D. Greer, and A. M. Okamura, "A soft robot that navigates its environment through growth," Sci. Robot., vol. 2, no. 8, p. eaan3028, 2017.

[8] D.-Y. Lee, S.-R. Kim, J.-S. Kim, J.-J. Park, and K.-J. Cho, "Origami Wheel Transformer: A Variable-Diameter Wheel Drive Robot Using an Origami Structure," Soft Robot., vol. 4, no. 2, pp. 163-180, jun 2017.
[9] V. Vitiello, S. L. Lee, T. P. Cundy, and G. Z. Yang, "Emerging robotic platforms for minimally invasive surgery," IEEE Rev. Biomed. Eng., vol. 6, pp. 111-126, 2013.

[10] K. Ikuta, H. Ichikawa, K. Suzuki, and T. Yamamoto, "Safety active catheter with multi-segments driven by innovative hydro-pressure micro actuators," Sixt. Annu. Int. Conf. Micro Electro Mech. Syst. 2003. MEMS-03 Kyoto. IEEE, pp. 130-135.

[11] B. Gorissen, M. De Volder, and D. Reynaerts, "Chip-on-tip endoscope incorporating a soft robotic pneumatic bending microactuator," Biomed. Microdevices, vol. 20, no. 3, p. 73, sep 2018.

[12] B. Gorissen, W. Vincentie, F. Al-Bender, D. Reynaerts, and M. De Volder, "Modeling and bonding-free fabrication of flexible fluidic microactuators with a bending motion," J. Micromech. Microeng, vol. 23, pp. $45012-10,2013$.

[13] L. Hines, K. Petersen, G. Z. Lum, and M. Sitti, "Soft Actuators for Small-Scale Robotics," Adv. Mater., vol. 29, no. 13, 2017.

[14] S. Konishi, S. Shimomura, S. Tajima, and Y. Tabata, "Implementation of soft microfingers for a hMSC aggregate manipulation system," Microsystems Nanoeng., vol. 2, no. November 2015, p. 15048, feb 2016.

[15] B. Gorissen, M. de Volder, and D. Reynaerts, "Pneumatically-actuated artificial cilia array for biomimetic fluid propulsion." Lab Chip, vol. 15 , no. 22 , pp. 4348-4355, 2015.

[16] K. Suzumori, S. Iikura, and H. Tanaka, "Development of flexible microactuator and its applications to $\backslash$ nrobotic mechanisms," Proceedings. 1991 IEEE Int. Conf. Robot. Autom., no. April, pp. 1622-1627, 1991.

[17] S. Konishi, F. Kawai, and P. Cusin, "Thin flexible end-effector using pneumatic balloon actuator," Sensors Actuators, A Phys., vol. 89, no. 1-2, pp. 28-35, 2001.

[18] Keiko Ogura, S. Wakimoto, K. Suzumori, and Yasutaka Nishioka, "Micro pneumatic curling actuator - Nematode actuator -," in 2008 IEEE Int. Conf. Robot. Biomimetics, 2009, pp. 462-467.

[19] J. Paek, I. Cho, and J. Kim, "Microrobotic tentacles with spiral bending capability based on shape-engineered elastomeric microtubes," Sci. Rep., vol. 5, no. March, p. 10768, 2015.

[20] M. De Volder, J. Coosemans, R. Puers, and D. Reynaerts, "Characterization and control of a pneumatic microactuator with an integrated inductive position sensor," Sensors Actuators, A Phys., vol. 141, no. 1, pp. 192-200, jan 2008.

[21] M. De Volder and D. Reynaerts, "Development of a hybrid ferrofluid seal technology for miniature pneumatic and hydraulic actuators," Sensors Actuators, A Phys., vol. 152, no. 2, pp. 234-240, jun 2009.

[22] B. Mosadegh, P. Polygerinos, C. Keplinger, S. Wennstedt, R. F. Shepherd, U. Gupta, J. Shim, K. Bertoldi, C. J. Walsh, and G. M. Whitesides, "Pneumatic networks for soft robotics that actuate rapidly," Adv. Funct. Mater., vol. 24, no. 15, pp. 2163-2170, 2014.

[23] E. Milana, B. Gorissen, D. Volder, and D. Reynaerts, "Design of a bi-segmented soft actuator with hardware encoded quasi-static inflation sequence," 2018 IEEE Int. Conf. Soft Robot., pp. 108-113, apr 2018.

[24] J. L. Sparks, N. A. Vavalle, K. E. Kasting, B. Long, M. L. Tanaka, P. A. Sanger, K. Schnell, and T. A. Conner-Kerr, "Use of silicone materials to simulate tissue biomechanics as related to deep tissue injury," Adv. Ski. Wound Care, vol. 28, no. 2, pp. 59-68, 2015.

[25] Smooth-On, "Dragon Skin ® Series," Smooth-On, 2015.

[26] J. Fleischer, T. Masuzawa, J. Schmidt, and M. Knoll, "New applications for micro-EDM," in J. Mater. Process. Technol., vol. 149, no. 1-3. Elsevier, jun 2004, pp. 246-249.

[27] T. Masuzawa, M. Fujino, K. Kobayashi, T. Suzuki, and N. Kinoshita, "Wire Electro-Discharge Grinding for Micro-Machining," CIRP Ann. - Manuf. Technol., vol. 34, no. 1, pp. 431-434, jan 1985.

[28] M. Bellotti, E. Milana, B. Gorissen, J. Qian, and D. Reynaerts, "Wire electrical discharge grinding of micro rods for bonding-free fabrication of soft pneumatic microactuators," European Society for Precision Engineering and Nanotechnology, Conference Proceedings - 18th International Conference and Exhibition, EUSPEN 2018, 2018. 